\title{
Design of Control System for Automatic Bamboo Splitting Equipment Based on PLC
}

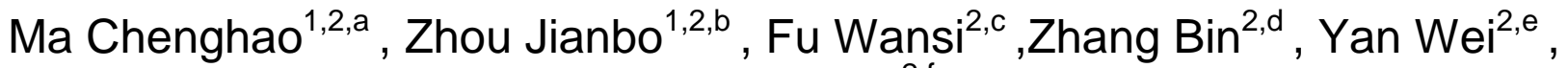 \\ Chang Feihu,
}

${ }^{1}$ Institute of New Forest Technology, Chinese Academy of Forestry, Beijing 100091, China

${ }^{2}$ Beijing Forestry Machinery Research Institute of the State Forestry Administration, Beijing 100029,

China

amachenghao36@163.com, ${ }^{\mathrm{b}}$ zhoujianbol@126.com, ${ }^{\mathrm{c}}$ fuwansi@126.com,

zhangbinbj1234@163.com, ${ }^{\mathrm{e}}$ 1337828220@qq.com, ${ }^{\mathrm{f}}$ cfh2016@126.com

Keywords: bamboo processing; Automatic; PLC

\begin{abstract}
Along with the increase of demand for bamboo, people has aroused the great importance to bamboo machinery automation, but now the low degree of the bamboo machine automation leading to the bamboo processing work is jointly by man-machine cooperation. This kind of excessive proportion of manual operation, cause the intensity of labor, repetitive movements, and highly security problem. To this end, this paper introduces a kind of automatic bamboo processing machine control system based on PLC, realizing the bamboo cutting and saving manpower, ensure the safety of personnel.
\end{abstract}

\section{Introduction}

With the development of social economy, the development of bamboo industry got more and more attention and a wide range of other social concerns. Bamboo possesses bamboo joint and hollow special structure. It owns the feature of breeding insect, moulding and seasoning crack, so both its processing technology and processing machinery, bamboo and wood are very different. Because of the limitation of technology and cost factors, existing bamboo machinery mainly transform directly from woodworking machinery. At present, the cutting tools of Bamboo Splitting Equipment (BSE) are selected mainly by workers eyes visual, and workers hold a bamboo tube on the cutting tool of BSE for stamping breaking bamboo, there is serious security hidden trouble. According to visual the diameter of the bamboo tube, workers replace the cutting tools. But workers' visual inaccuracy could do a lot of waste. In order to improve these problems, developers with more attention paid to the development of $\mathrm{BSE}^{[1]}$.

This paper introduces a kind of BSE control system based on PLC, including feeding and tool change work dangerously are completed by the control system operation. It effectively save manpower and ensure the staff's personal safety.

\section{Technology Introduction of PLC}

Programmable Logic Controller, hereinafter referred to as PLC, is a kind of general-purpose automatic control device based microprocessor. It usually gives priority to with sequence control and loop adjustment is complementary, can accomplish logic, sequence, timing, counting and arithmetic operations, and other functions, can not only control switch quantity, also can control analog. In recent years, PLC technology develops rapidly, many new products are launched every year. PLC is essentially a kind of industrial control dedicated computer, PLC system with microcomputer structure is basically the same, is composed of hardware system and software system of two parts. General-purpose PLC hardware structure is shown in figure 1, it is a universal programmable logic controller, mainly by the central processing unit (CPU), memory, I/O module and power supply ${ }^{[2]}$. 


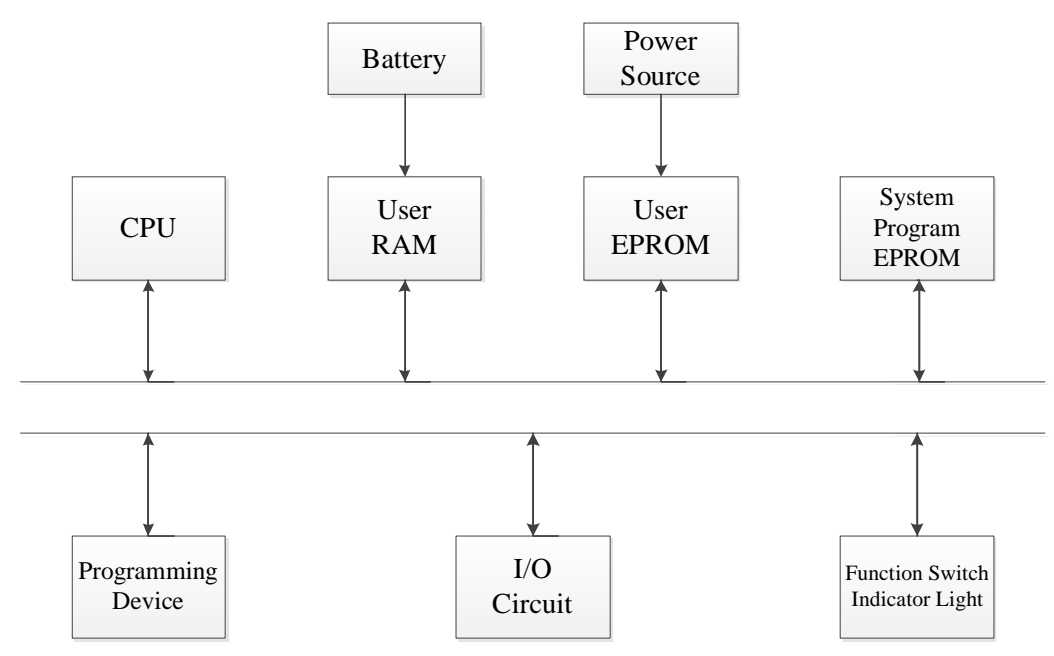

Fig.1 General-purpose PLC hardware structure

This automatic broken bamboo machine adopts PLC control system, good at using PLC sequence control, better on the various steps of BSE to carry out accurate operation, so as to realize the automation of BSE.

\section{Control System}

\subsection{System Summary}

BSE's main parts as follows: bamboo transmission device, the tool dish and bamboo fixtures, its equipment diagram is shown in figure 2.

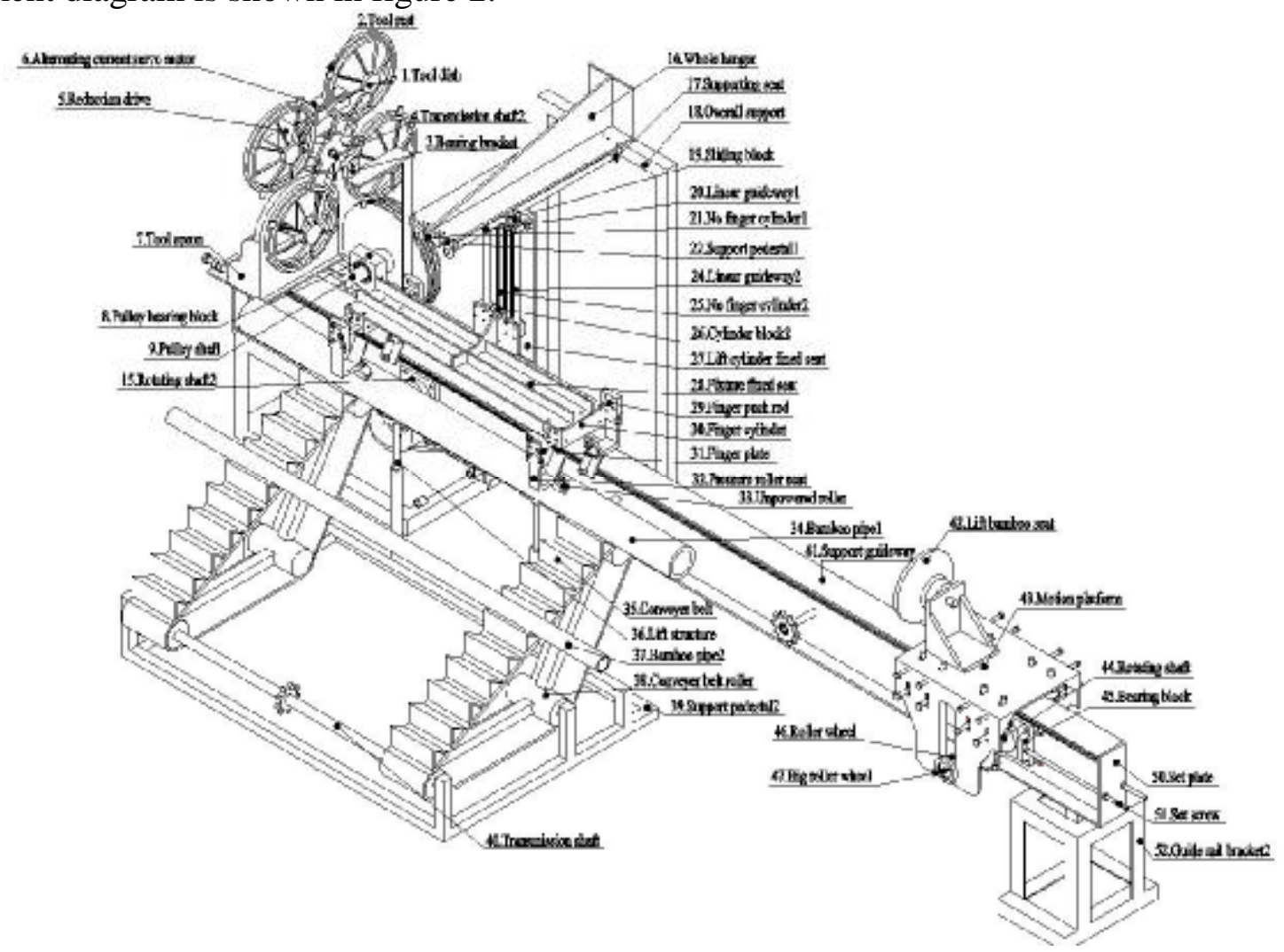

Fig. 2 BSE equipment

1.tool dish 、2.tool rest、3.bearing bracket、4.transmission shaft2、5.reduction drive、6.alternating current servo motor、7.tool apron、8.pulley bearing block、9.pulley shaft、10.big pulley、11.guide rail bracket1、12.small pulley、13.motor、14.motor mount、15.rotating shaft 2 16.whole hanger、 
17.supporting seat、18.overall support、19.sliding block、20.linear guideway1 、21.no finger cylinder1、22.support pedestal1 、23.cylinder block1、24.linear guideway2、25.no finger cylinder2、 26.cylinder block2、27.Lift cylinder fixed seat、28.fixture fixed seat、29.finger push rod、30.finger cylinder、31.finger plate 、32.pressure roller seat、33. unpowered roller、34.bamboo pipe1、35.conveyer

belt 、36.lift structure、37.bamboo pipe2、38.conveyer belt roller、39.support pedestal2、 40.transmission shaft、41.support guideway、42.lift bamboo seat、43.motion platform、44.rotating shaft、45.bearing block、46.roller wheel、47.big roller wheel、48.reactro、49.hold chain block、

50.set plate、51.set screw、52.guide rail bracket2

The control system is mainly divided into the following several parts: bamboo tube feeding, blade choice, bamboo centering, bamboo cutting. Bamboo tube feeding namely bamboo timber transport to the designated cutting position, and choose system specified by the blade on the tool dish blade model, finally by a central system aim the bamboo center and the center of the blades, for cutting. The main work process is shown in figure 3.

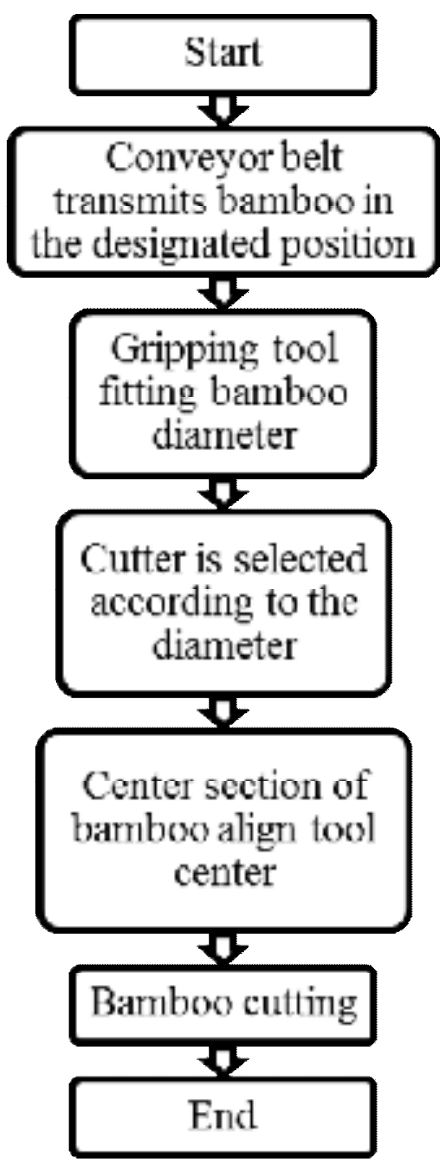

Fig. 3 Flow chart of control system

Described BSE automatic switch cutter device can be mounted to the rest of 4 tool dish, tool plate can be installed on the number of the blade is $12 \sim 15$. Bamboo diameter that can be processed in the range of $60 \sim 120 \mathrm{~mm}$. The gripping device can grab the bamboo for $2 \sim 2.5 \mathrm{~kg}$, the length of 1500 $\sim 2500 \mathrm{~mm}$, in line with the general bamboo processing needs.

\subsection{Bamboo Tube Feeding System}

Bamboo tube feeding system performed by ladder type conveyor belt. Bamboo tube feeding system performed by ladder type conveyor belt. Using PLC control motor speed and pause, bamboo was sent to cutting machine. Trapezoidal conveyor belt, compared with other transmission device has the advantages of simpleness and speediness, it can use more simple control process will transfer 
bamboo timber to the specified location, to deliver goods, speed automatically according to the distance between the two items can transform to prevent collision between the articles; can realize fault alarm, status indication, the conveyor belt load soft start, etc.; to realize automatic and manual state switch, convenient maintenance. The system mainly uses the PLC, sensors, relays, inverter and other components, good automatic control performance by using PLC, to realize to control by no one in the process of assembly line conveyor belt transmission ${ }^{[3]}$. The main control process is shown in figure 4 .

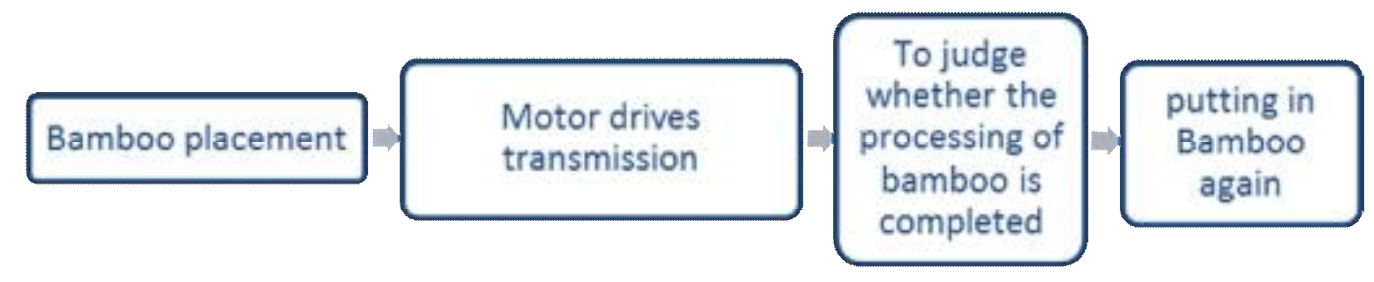

Fig. 4 Bamboo tube feeding control process

\subsection{Blade Selection System}

Blade selection system is the most crucial part of the machine control system among the whole automatic BSE, at present most of the semi-automatic BSE mainly need to solve the problem. Due to the different diameter of bamboo or demand purpose, bamboo is often need to be processed into different size materials. Method is general by manual operation, namely estimate its diameter by staff visual bamboo, artificially turn the tool dish, then cut bamboo. This method is not only a waste of resources, easy to cause error, to threaten the safety of operating personnel importantly.

This system adopted the control mode of automatic measurement of bamboo diameter and the choice of tool, in order to overcome this problem. Bamboo fixed grab section of the cutting table is shown in figure 5 .

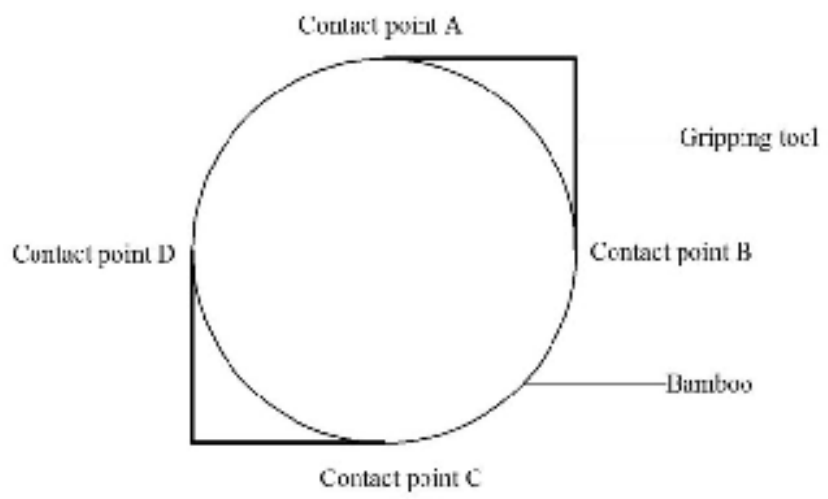

Fig. 5 Bamboo grab interface

From the above, we can see that there are 4 contact points between the bamboo grasp tool and bamboo, and the spatial position of the 4 contact points can be obtained by using the sensor. Because the cross section of bamboo are not regularly circular, this would require the fitting method, get the fitting the diameter of the circle, as the parameter compared with 4 cutter diameter of cutter head on, to get the suitable cutting tool ${ }^{[4]}$.

In order to get the fitting circle, the plane fitting of the contact point should be carried out firstly. Since all the observation points must be on the plane, it is necessary to carry out the plane fitting of the observation points. Any space plane equation can be represented as: 


$$
\mathrm{ax}+\mathrm{by}+\mathrm{cz}-1=0
$$

The 4 observation points can be brought into the above formula.

$$
\mathrm{A} \cdot \mathrm{X}-\mathrm{L}=0
$$

$$
\mathrm{A}=\left[\begin{array}{cccc}
\mathrm{x}_{1} & \mathrm{x}_{1} & \mathrm{x}_{1} & \mathrm{x}_{1} \\
\mathrm{y}_{1} & \mathrm{y}_{1} & \mathrm{y}_{1} & \mathrm{y}_{1} \\
\mathrm{z}_{1} & \mathrm{z}_{1} & \mathrm{z}_{1} & \mathrm{z}_{1}
\end{array}\right]^{\mathrm{T}} \quad, \quad \mathrm{X}=(\mathrm{a}, \mathrm{b}, \mathrm{c})^{\mathrm{T}}, \mathrm{L}=(1,1,1,1)^{\mathrm{T}}
$$

weight matrix $\mathrm{P}$ is A unit matrix.

In this way, according to the least square rule $\mathrm{V}^{\wedge} \mathrm{T} P V=\min$, the direction coefficient of the fitting plane's normal vector is:

$$
X^{\prime}=\left(A^{T} A\right)^{-1} A^{T} L
$$

And the various points $\mathrm{P}\left(\mathrm{x} \_\mathrm{i}, \mathrm{y} \_\mathrm{i}, \mathrm{z} \_\mathrm{i}\right)$ flatness:

$$
\mathrm{D}_{\mathrm{i}}=\left|\frac{\mathrm{ax}+\mathrm{by}+\mathrm{cz}-1}{\sqrt{\mathrm{a}^{2}+\mathrm{b}^{2}+\mathrm{c}^{2}}}\right|,(i=1,2,3 \ldots)
$$

4 observation points are fitted to the same plane, and the fitting of the plane circle can be carried out. In the space circle between arbitrary points can be a string, the linear equation of the string calculated the corresponding vertical plane equation, measurement point $\mathrm{N}(\mathrm{N}>3)$ as an example, we can get N-1 linear irrelevant strings, so that in the vertical plane of a linear equation is not related to $\mathrm{N}-1$. According to the characteristics of space circle, fitting plane and all space plane intersection and only one intersection, the intersection is the center of the plane circle. Finally, the space plane fitting the restriction will be as the limiting condition, the vertical plane is used as the observation equation, by the indirect adjustment with constraints as basic function model to derive the calculation equations of plane circle, according to point to the center of the algebraic distance equation calculate plane circle diameter ${ }^{[5]}$.

\subsection{Bamboo Tube Alignment System}

The automatic centering device is a device which is used for positioning and clamping at the same time, and is used for the occasions that the center line and the symmetrical plane are used as the working procedures.

It can be divided into two types.

1.According to the uniform motion or rotation principle of the positioning and clamping elements, the system realizes the centering and clamping.

2.According to the uniform elastic deformation principle of the positioning and clamping elements, the system realizes the centering and clamping.

Between the integrity of bamboo and the clamping force of grab the tool have a close relationship.

The principle of determining the size of the clamping force is that when the bamboo is clamped, the accurate positioning of the bamboo can't be destroyed, and the damage of the bamboo after the clamping deformation and the compression surface is not allowed to exceed the range of the technical conditions. The clamping force directly affects the processing reliability, the clamping deformation, the positioning accuracy and the processing precision of bamboo. In actual machining process, there are many factors that affect the clamping force, and the calculation is very complicated. In the strict sense, the clamping force is a rough estimate of value. On the basis of the principle of static balance, the force sensor on the grasping tool plays an important role ${ }^{[6]}$. 
Because of the space fitting circle have been completed, can easily find the spatial coordinates of the bamboo tube fitting section circle cross, with constant speed movement principle of clamping device can realize automatic clamping action. Using the grab tool and the position sensor of the cutting table, the center of the bamboo and the center of the blade can be aligned. After the alignment is finished, the pressure sensor of the grab tool reaches the threshold. In the end, the bamboo is fed into the blade by the cutting table and the grasping tool, and the automatic cutting of the bamboo is finished. At this point, the work flow of automatic BSE is completed. The automatic BSE control system, which is controlled by PLC, has completed the most difficult blade selection system, saves manpower and material resources, and ensures the personal safety of the staff ${ }^{[7]}$.

\section{Conclusions}

In this paper, based on the widely used semi automatic BSE low work efficiency, safety and other shortcomings, puts forward the control scheme of BSE, widely used in sequence control by using PLC technology, the automation of the manual tool change of BSE, so as to realize the automation of the whole machining process of BSE. To reduce the error caused by the artificial visual tool change, tool change to avoid security risks in the process, and save manpower cost, is of great significance for the production of life.

\section{Acknowledgements}

This work was financially supported by the Research on common key technology and equipment of bamboo primary processing(2016SY10).

\section{Literature References}

[1] Zhang Jing, Huang Qin, Lan Hongjun. Spatial circle fitting method in engineering survey research [J]. Metrology \& Measurement Technique, 2011, 38(9): 31-32. (in Chinese)

[2] Pan Guorong, Chen xiaolong. Method of fitting spatial circular objects [J]. Journal of Geodesy and Geodynamics, 2008, 28(2): 92-94. (in Chinese)

[3] Cui TX, Lv RT, Kang FY et a1. Synthesis and enhanced field-emission of thin-walled, open-ended, and wen-aligned N-doped carbon nanotubes [J]. Nanoscale R. es Lett. 2010.

[4] Zhao Renjie. Technology of artificial bamboo board [M]. Beijing: China Forestry Publishing House, 2002. (in Chinese)

[5] Yan Yonglin. Design conception of the inner section machine [J]. Forestry Machinery \& Woodworking Equipment, 2000(1): 13-14. (in Chinese)

[6] Zhang Qisheng. Industrial utilization of bamboo in China [M]. Beijing: China Forestry Publishing House, 1999. (in Chinese)

[7] Xia Luosheng. Application of PLC in fault diagnosis of CNC machine tools [J]. Machine Tool Electric Apparatus, 2006. (in Chinese) 